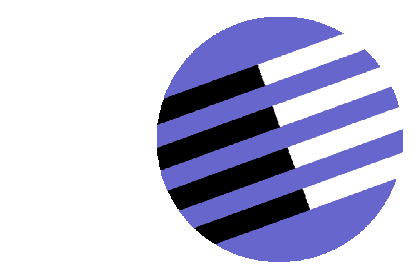

GOVERNANCE AND THE EFFICIENCY

OF ECONOMIC SYSTEMS

G ES Y

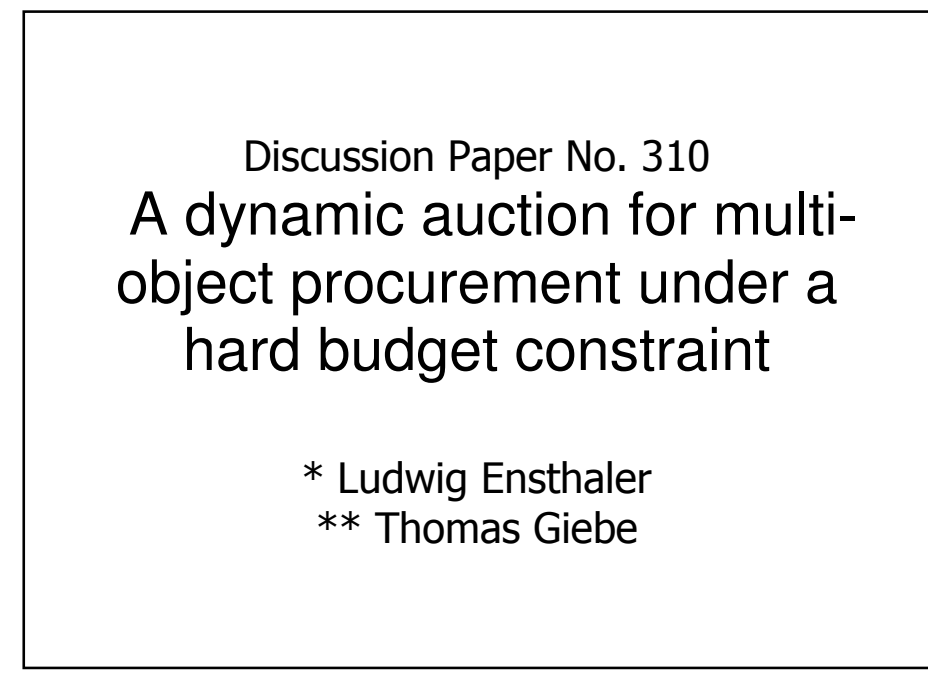

* Humboldt University at Berlin

** Humboldt University at Berlin

March 2010

Financial support from the Deutsche Forschungsgemeinschaft through SFB/TR 15 is gratefully acknowledged. 


\title{
A dynamic auction for multi-object procurement under a hard budget constraint*
}

\author{
Ludwig Ensthaler ${ }^{\dagger}$ \\ Thomas Giebe ${ }^{\ddagger}$ \\ Humboldt University at Berlin \\ DIW Berlin \\ Humboldt University at Berlin
}

March 3, 2010

\begin{abstract}
We present a new dynamic auction for procurement problems where payments are bounded by a hard budget constraint and money does not enter the procurer's objective function.
\end{abstract}

JEL D21, D44, D45, D82.

Keywords: Auctions, Mechanism Design, Knapsack Problem, Dominant Strategy, Budget, Procurement

\footnotetext{
* Many thanks to Ulrich Kamecke, Roland Strausz, Balázs Szentes, Georg Weizsäcker, and Elmar Wolfstetter for helpful comments. Financial support from the Deutsche Forschungsgemeinschaft through SFB/TR 15 is gratefully acknowledged.

${ }^{\dagger}$ Humboldt University at Berlin, School of Business and Economics, Dept. of Economics, Spandauer Straße 1, 10178 Berlin, Germany, and Graduate Center of Economic and Social Research DIW Berlin, Tel: +49-30-2093-1594, Fax: +49-30-2093-5619, lensthaler@diw.de

${ }^{\ddagger}$ (Corresponding author) Humboldt University at Berlin, School of Business and Economics, Dept. of Economics, Spandauer Straße 1, 10178 Berlin, Germany, Tel: +49-302093-5773, Fax: +49-30-2093-5619, thomas.giebe@wiwi.hu-berlin.de.
} 


\section{INTRODUCTION}

A budget-constrained buyer wants to purchase items from a shortlisted set. Items are differentiated by observable quality and sellers have private reserve prices for their items. Sellers quote prices strategically, inducing a knapsack game. The buyer's problem is to procure a subset of maximal quality. We study a procurement auction that can be viewed as a game-theoretic extension of the greedy-split heuristic for the classic knapsack problem. The auction exhibits truthtelling in dominant strategies, is ex-post rational, and satisfies the hard budget constraint. We see many applications for this procurement setting, e.g., allocating R\&D subidies, scholarships, emission reductions.

Despite its relevance, the problem has received surprisingly little attention in the literature. We introduced the auction in Ensthaler and Giebe (2009). In independent research, approaching the problem from a different angle, Papadimitriou and Singer (2010) analyze a similar direct mechanism in a more general framework. The allocation problem was first studied by Giebe et al. (2006) in the context of R\&D subsidies. Chung and Ely (2002) (extending the work of Maskin, 2002) derive the optimal Bayesian mechanism for a similar procurement problem under a soft budget constraint.

\section{THE MODEL}

Let $\mathcal{I}:=\{1, \ldots, N\}, N \geq 1$, denote the set of sellers where $i$ is a typical seller. Each seller wants to sell an indivisible item for which he has a privately known reserve price $\theta_{i}$. Items differ in quality and quality is common knowledge. Let $w_{i}>0$ denote the quality of seller $i$ 's item. Sellers are faced by a single buyer who has a fixed and finite budget $\mathcal{B}$ to spend on these items. The buyer's objective is to purchase a subset of items of maximal quality, where the quality of a set of items is the sum of qualities of its elements. Money does not enter the buyer's utility function. Sellers' types satisfy $\theta_{i} \in[0, \mathcal{B}]$. We assume that the probability of two types being equal is zero and that there is no known statistical relation between sellers' types, resp. between quality and types.

In the following, a mechanism is an open auction or a direct mechanism. We restrict attention to symmetric deterministic mechanisms, where the sellers submit bids $b_{i} \geq 0$ (or type reports $b_{i} \in[0, \mathcal{B}]$ ). The vector of sellers' bids (reports) is $b=\left(b_{i}, b_{-i}\right)=\left(b_{1}, \ldots, b_{N}\right)$ where a truthful report is $b_{i}=\theta_{i}$. For given bids, $b$, and true type $\theta_{i}$, seller $i$ 's ex-post utility is

$$
u_{i}\left(b \mid \theta_{i}\right):=t_{i}(b)-q_{i}(b) \theta_{i},
$$

where $t_{i}$ is a payment to $i$ and $q_{i} \in\{0,1\}$. Let $\mathcal{A}(b):=\left\{i \in \mathcal{I} \mid q_{i}(b)=1\right\}$ denote the allocation resulting from the play of a mechanism when the vector of final bids (type reports) is $b$, and denote the complementary set by $\mathcal{A}^{c}$. 
The allocation is the set of sellers whose objects are procured. For simplicity, denote $u_{i}(b):=u_{i}\left(b \mid \theta_{i}\right)$ and $\mathcal{A}:=\mathcal{A}(b)$ if confusion is unlikely.

The buyer's utility is the total quality of the procured items,

$$
u_{\text {buyer }}(\mathcal{A})=\sum_{j \in \mathcal{A}} w_{j}=\sum_{i \in \mathcal{I}} q_{i} w_{i}, \quad q_{i} \in\{0,1\} .
$$

Under complete information, the buyer faces a simple knapsack problem,

$$
\arg \max _{q} \sum_{i \in \mathcal{I}} w_{i} q_{i} \text { s.t. } \sum_{i \in \mathcal{I}} \theta_{i} q_{i} \leq \mathcal{B}, q \in\{0,1\}^{N} .
$$

Call (3) the first-best allocation(s).

\section{THE AUCTION}

Consider the following open descending clock auction. A central continuous clock counts down the price-per-quality ratio $r$, beginning from the highest initial ratio among all bidders, $r^{\max }:=\max _{i \in \mathcal{I}} \mathcal{B} / w_{i}$. A bidder can quit the auction at any time, but can never come back. Being active at clock reading $r$ implies that bidder $i$ is willing to accept a payment of $r w_{i}$ in return for his item.

As the clock counts down, each active bidder's current financial bid, $r w_{i}$, decreases. As long as the sum of active financial bids exceeds the budget, the countdown continues. As soon as that sum fits in the budget, say, at round $r^{f}$, the auction ends, and each active bidder $i$ sells his item at the price $r^{f} w_{i}$.

The sum of active financial bids decreases smoothly as the clock counts down, until some bidder $j$ exits, at which point that sum is reduced by the amount $r w_{j}$.

Two events can end the auction. First, the end can be triggered by an exit, when before the exit, the sum of active financial bids exceeded the budget, but after the exit the remaining active bids can be accomodated without exhausting the budget. Second, it may end because at some point all active bids exactly use up the budget.

After the auction, each loser $j$ has a final ratio $r_{j}=b_{j} / w_{j}$ with financial bid $b_{j}$ since $j$ quit the auction at clock reading $r=r_{j}$. All winners $i$ have the same final ratio $r^{f}=b_{i} / w_{i}$ with (different) selling prices $b_{i}$.

Lemma 1. The stop rule $b_{i}\left(\theta_{i}\right)=\theta_{i}$ is a weakly dominant strategy.

Proof. Suppose a seller plays the candidate. His profit is either zero, if he has to quit before the auction ends, or it is positive, if the auction ends before he quits. Consider stopping earlier. Either there is no change or he quits but could have made a profit. Consider stopping later. Either there is no change, or he makes a loss if the auction ends after $r=\theta_{i}$ but before he quits. 
In order to give a direct characterization of the auctions' equilibrium result we need the following notation.

Let $l_{i}$ denote the set of players with final ratio not above that of bidder $i$. Let $r_{\mathcal{A}^{c}}$ denote the smallest ratio among all players who are not in the allocation (unless $\mathcal{A}^{c}=\emptyset$ ).

$$
\begin{aligned}
r_{i} & :=\left\{j \in \mathcal{I} \mid \frac{b_{j}}{w_{j}} \leq \frac{b_{i}}{w_{i}}\right\} \\
r_{\mathcal{A}^{c}} & := \begin{cases}\min _{\mathcal{B}}\left\{\frac{b_{j}}{w_{j}} \mid j \in \mathcal{A}^{c}\right\} & \text { if } \mathcal{A}^{c} \neq \emptyset \\
\frac{\operatorname{Bin}_{j \in \mathcal{I}} w_{j}}{\operatorname{li}_{j}} & \text { if } \mathcal{A}^{c}=\emptyset\end{cases}
\end{aligned}
$$

Lemma 2. In equilibrium $\left(b_{i}=\theta_{i}\right)$, the auction implements allocation $\mathcal{A}$ and payments $t$ where

$$
\begin{aligned}
q_{i}(b) & = \begin{cases}1 & \text { if } \frac{b_{i}}{w_{i}} \sum_{j \in l_{i}} w_{j} \leq \mathcal{B} \\
0 & \text { otherwise },\end{cases} \\
t_{i}(b) & = \begin{cases}\min \left\{r_{\mathcal{A}^{c}} w_{i}, \frac{\mathcal{B}}{\sum_{j \in \mathcal{A}} w_{j}} w_{i}\right\} & \text { if } q_{i}(b)=1 \\
0 & \text { otherwise. }\end{cases}
\end{aligned}
$$

Proof. By Lemma 1, winners' true ratios are below the final ratio, $\theta_{i} / w_{i}<$ $r^{f}=b_{i} / w_{i}$ since they were active at the end of the auction. Thus, winners have lower true ratios than losers. All winners are paid according to the same ratio, $r^{f}$, i.e. winner $i$ gets $b_{i}=r^{f} w_{i}$. Thus, $i$ is a winner iff it is feasible to pay every player with same or lower final ratio according to $i$ 's final ratio, see (6).

Losers receive no payment and keep their items. The payments to the winners $i \in \mathcal{A}$ depend on which event ends the auction. If the end is triggered by an exit, then the exiting player's final ratio determines the payments, $r_{\mathcal{A}^{c}} w_{i}$, otherwise payments are $\frac{\mathcal{B}}{\sum_{j \in \mathcal{A}} w_{j}} w_{i}$, i.e., they use up the budget.

The second line in (5) just makes the definition complete but is never payoffrelevant.

Observation 1. Suppose $b_{i} \in[0, \mathcal{B}]$ are type reports. Then (6) and (7) define an incentive-compatible direct mechanism $(q, t)$ with allocation and payments as in the auction. This mechanism exhibits truthtelling in dominant strategies, satisfies the hard budget constraint and is ex-post individually rational.

\section{DISCUSSION}

Obviously, the auction observes the hard budget constraint. It is ex-post rational since all winners are paid according to a ratio that exceeds their true ratio and losers pay nothing. 
Apart from these appealing incentive properties, how well does the auction perform compared to the first-best solution?

For the special case where all items have the same quality, one can easily show that the auction is a 2-approximation, which means that in every instance, the first-best allocation is at most twice as good as the allocation chosen by the auction.

For the general case with different qualities Papadimitriou and Singer (2010) show that a slightly modified direct mechanism is a 6-approximation.

Though these worst-case bounds may not sound encouraging, simulations indicate that the average performance of the auction is likely to be much better. ${ }^{1}$

Is there a mechanism that is better or optimal (on average)? We see plenty of room for further research.

\section{REFERENCES}

Chung, K.-S. And J. C. Ely (2002): "Mechanism Design with Budget Constraint," Working Paper.

Ensthaler, L. And T. Giebe (2009): "Subsidies, Knapsack Auctions and Dantzig's Greedy Heuristic," SFB/TR 15 Discussion Paper No. 254.

Giebe, T., T. Grebe, and E. Wolfstetter (2006): "How to allocate R\&D (and other) subsidies: An experimentally tested policy recommendation," Research Policy, 35, 1261-1272.

MAskin, E. (2002): "How to reduce greenhouse gas emissions - an application of auction theory," Nancy L. Schwartz Memorial Lecture, Northwestern University.

Papadimitriou, C. And Y. Singer (2010): "Budget Feasible Mechanisms," Working Paper (University of California at Berkeley).

\footnotetext{
${ }^{1}$ See Ensthaler and Giebe (2009).
} 